\title{
Global invariants of paths and curves for the group of orthogonal transformations in the two-dimensional Euclidean space
}

\author{
Djavvat Khadjiev and İdris Ören*
}

\begin{abstract}
In this paper, for the orthogonal group $G=O(2)$ and special orthogonal group $G=O^{+}(2)$ global $G$-invariants of plane paths and plane curves in two-dimensional Euclidean space $E_{2}$ are studied. Using complex numbers, a method to detect $G$-equivalences of plane paths in terms of the global $G$-invariants of a plane path is presented. General evident form of a plane path with the given $G$-invariants are obtained. For given two plane paths $x(t)$ and $y(t)$ with the common $G$-invariants, evident forms of all transformations $g \in G$, carrying $x(t)$ to $y(t)$, are obtained. Similar results have obtained for plane curves.
\end{abstract}

\section{Introduction}

Transformations and invariants of curves, surfaces and graphical objects appear in many areas of Computer Aided Geometric Design, Computer Aided Design, computer graphics, computer vision and pattern recognition. One of the most important problems in computer vision and vision-based applications is the automatic identification of objects. Plane curves are fundamental objects in differential geometry. In industrial shape design or computer aided geometric design, curves of particular property have been used as a parts of

Key Words: Curve, Invariant, Euclidean geometry

2010 Mathematics Subject Classification: Primary 53A04; Secondary 53A55.

* Corresponding author

Received: 06.04.2018

Accepted: 30.06.2018 
figures (see in some references $[1,6,7,10,11,18]$ and therein references). The important problem is to find simple but efficient method for the equivalence check of two curves. The present paper is devoted to the global differential geometry of paths and curves in two-dimensional Euclidean space $E_{2}$ for the two-dimensional orthogonal group $O(2)$ and the special orthogonal group $O^{+}(2)=\{g \in O(2) \mid \operatorname{det}(g)=1\}$.

Below we use following definitions of a path, an $L$-regular path, a curve, a $G$-equivalence of paths and a $G$-equivalence of curves in the 2-dimensional Euclidean space $E_{2}$.

Let $R$ be the field of real numbers and $T=(a, b)$ be an open interval in $R$.

Definition 1.1. A $C^{(1)}$-mapping $x: T \rightarrow E_{2}$ will be called a $T$-path (parametrized curve) in $E_{2}$.

Let $G=O(2)$ or $G=O^{+}(2)$.

Definition 1.2. $T$-paths $x(t)$ and $y(t)$ in $E_{2}$ are called $G$-equivalent if there exists $F \in G$ such that $y(t)=F x(t)$. This being the case, we write $x(t) \stackrel{G}{\sim} y(t)$

Definition 1.3. A function $f(x(t), y(t), \ldots, z(t))$ of a finite number of $T$ paths $x(t), y(t), \ldots, z(t))$ is called $G$-invariant if $f(F x(t), F y(t), \ldots, F z(t))=$ $f(x(t), y(t), \ldots, z(t))$ for all $F \in G$, for all $T$-paths $x(t), y(t), \ldots, z(t)$ and for all $t \in T$.

Definition 1.4. A $T$-path $x(t)$ in $E_{2}$ will be called $L$-regular if $x(t) \neq 0$ for all $t \in T$.

Let $J_{1}=(a, b) \subseteq R$ and $J_{2}=(c, d) \subseteq R$.

Definition 1.5. (see $[4,15])$ A $J_{1}$-path $x(t)$ and a $J_{2}$-path $y(r)$ in $E_{2}$ will be called $D$-equivalent if a $C^{(1)}$-diffeomorphism $\varphi: J_{2} \rightarrow J_{1}$ exists such that $\varphi^{\prime}(r)>0$ and $y(r)=x(\varphi(r))$ for all $r \in J_{2}$. A class of $D$-equivalent paths in $E_{2}$ will be called a curve (non-parametrized curve) in $E_{2}$. A path $x \in \alpha$ will be called a parametrization of a curve $\alpha$.

Let $\alpha=\left\{h_{\tau}, \tau \in \Gamma\right\}$ be a curve in $E_{2}$, where $h_{\tau}$ is a parametrization of $\alpha$. Then $F \alpha=\left\{F h_{\tau}, \tau \in \Gamma\right\}$ is a curve in $E_{2}$ for any $F \in O(2)$.

Definition 1.6. (see $[4,15]$ ) Two curves $\alpha$ and $\beta$ in $E_{2}$ are called $G$-equivalent if $\beta=F \alpha$ for some $F \in G$. This being the case, we write $\alpha \stackrel{G}{\sim} \beta$.

Definition 1.7. A curve $\alpha$ will be called L-regular if it contains an L-regular path.

Remark 1.8. There are some definitions of specific curves available, related to the subject of our research, given by [2], [9, p.2], [12, p.25], [17, p.20-22]. 
For regular curves in classic differential geometry, the following theorem is known for the group $M^{+}(2)$ : "Let $\alpha$ and $\beta$ be two curves in the twodimensional Euclidean space $E_{2}$. Then, $\alpha$ and $\beta$ are $M^{+}(2)$-equivalent if and only if the curvatures and speeds of $\alpha$ and $\beta$ are equal", where $M^{+}(2)=\{F$ : $\left.E_{2} \rightarrow E_{2} \mid F x=g x+b, g \in O^{+}(2), b \in E_{2}\right\}$.

This theorem shows that a necessary and sufficient condition for two curves in $E_{2}$ to be $M^{+}(2)$-equivalent is that they have the same curvature and speed. Using method of Frenet frames of two curves, this theorem shows how to compute explicitly an isometry carrying $\alpha$ to $\beta$ (see [19]). In addition, the method give conditions only for the local $M^{+}(2)$-equivalence of curves.

Generally, in the classical differential geometry of curves in the $n$-dimensional Euclidean space $E_{n}$, where $n \geq 3$, using Frenet-Serret equations, curvature functions $k_{i}, i=1, \ldots, n-1$, of the curve were obtained (see [3, p.172]). The function $k_{i}$ is $M(n)$-invariant for $i=1, \ldots n-2$, where $M(n)=\left\{F: E_{n} \rightarrow E_{n} \mid F x=g x+b, g \in O(n), b \in E_{n}\right\}, O(n)$ is the group of all orthogonal transformations of $E_{n}$. But $k_{n-1}$ is not $M(n)$-invariant. It is $M^{+}(n)$-invariant, where $M^{+}(n)=\left\{F: E_{n} \rightarrow E_{n} \mid F x=g x+b, g \in O^{+}(n)\right.$, $\left.b \in E_{n}\right\}, O^{+}(n)=\{g \in O(n) \mid \operatorname{det}(g)=1\}$ is the group of all special orthogonal transformations of $E_{n}$. Thus invariant theory of curves in the classical differential geometry was developed only for the group $M^{+}(n)$. In addition, the method of orthogonal frame in the classical differential geometry give conditions only for the local $M^{+}(n)$-equivalence of curves.

In works $[4,12]$, by using invariant parametrization of curves, the problem of global $G$-equivalence of curves (that is nonparametrized curves) was reduced to the problem of global $G$-equivalence of paths (that is parametrized curves) for groups $G=M(n), M^{+}(n)$. Complete systems of global invariants of non-degenerate paths and non-degenerate curves in classical geometries were obtained. Moreover, systems of generators of differential field of $G$-invariant differential rational functions of a path in $E_{n}$ for groups $M(n)$ and $M^{+}(n)$ are obtained . This approach was developed for curves in papers [15, 16, 21, 22] and for vector fields in $[13,14]$.

In paper [20], an invariant-theoretic approach to problems of G-equivalence of Bézier curves for groups $G=M^{+}(n), M(n)$ is given. These papers contain solutions of problems of global G-equivalence of Bézier curves for these groups without using differential invariants of a Bézier curve.

The present paper is devoted to an investigation of problems of global $G$-equivalence of regular and non-degenerate paths in $E_{2}$ for groups $G=$ $O(2), O^{+}(2)$ in terms of global $G$-invariants of paths. For non-degenerate curves, this theory is also investigated. For solutions of these problems, we use complex numbers.

In the book [8, Theorems 6.1, 6.8], for the group $M^{+}(2)$, existence and 
uniqueness theorems for regular parametric curves in $E_{2}$ were obtained by using complex numbers. In the book [24], different concrete classes of plane curves investigated by using complex numbers. In the end of this book, "Historical notes" on the development of the concept of the complex number and applications of complex numbers are given. Some applications of three types of general complex numbers (ordinary complex numbers, hyperbolic numbers and dual numbers) to two-dimensional geometries are given in the book [23].

This paper is organized as follows. In Sec. 2, some known results on descriptions of groups $G=O(2)$ and $O^{+}(2)$ in terms of complex numbers are given. The fundamental $G$-invariants of a path in $E_{2}$ for the groups $G=$ $O(2), O^{+}(2)$ are given. In Sec. 3, complete systems of global $G$-invariants of a regular path and uniqueness theorems for regular paths are given. In Sec. 4, theorems on an existence for regular paths and evident forms of a path with given $G$-invariants are obtained. In Sec. 5, the type and invariant parametrizations of an $L$-non-degenerate curve are defined and investigated. In Sec. 6, complete systems of $G$-invariants of an L-non-degenerate curve and uniqueness theorems for L-non-degenerate curves are obtained.

\section{The groups $G=O(2), O^{+}(2)$ and fundamental $G$-invariants of a path in $E_{2}$}

Let $R$ be the field of real numbers and $\Omega$ be the field of complex numbers. The multiplication in $\Omega$ has the form $\left(a_{1}+i a_{2}\right)\left(b_{1}+i b_{2}\right)=\left(a_{1} b_{1}-a_{2} b_{2}\right)+i\left(a_{1} b_{2}+\right.$ $\left.a_{2} b_{1}\right)$. We will consider element $a=a_{1}+i a_{2}$ also in the form $a=\left(\begin{array}{c}a_{1} \\ a_{2}\end{array}\right)$. For $a=a_{1}+i a_{2}$, denote by $P_{a}$ the matrix $\left(\begin{array}{cc}a_{1} & -a_{2} \\ a_{2} & a_{1}\end{array}\right)$ and consider $P_{a}$ also as the transformation $P_{a}: \Omega \rightarrow \Omega$, where $P_{a} b=\left(\begin{array}{cc}a_{1} & -a_{2} \\ a_{2} & a_{1}\end{array}\right)\left(\begin{array}{l}b_{1} \\ b_{2}\end{array}\right)=$

$$
\begin{aligned}
\left(\begin{array}{c}
a_{1} b_{1}-a_{2} b_{2} \\
a_{1} b_{2}+a_{2} b_{1}
\end{array}\right) \text { for } b=b_{1}+i b_{2} & =\left(\begin{array}{l}
b_{1} \\
b_{2}
\end{array}\right) . \text { Then we have the equality } \\
a b & =P_{a} b .
\end{aligned}
$$

for all $a, b \in \Omega$. Let $P(\Omega)$ denote the set of all matrices $P_{a}$, where $a \in \Omega$. We consider on $P(\Omega)$ the following standard matrix operations: the componentwise addition, a scalar multiplication and the multiplication of matrices. Then $P(\Omega)$ is a field, where the unit element is the unit matrix. The following Propositions 2.1-2.5 are known.

Proposition 2.1. The mapping $P: \Omega \rightarrow P(\Omega)$, where $P: a \rightarrow P_{a}$ for all $a \in \Omega$, is an isomorphism of fields. 
For vectors $a=a_{1}+i a_{2}, b=b_{1}+i b_{2} \in \Omega$, we put $\langle a, b\rangle=a_{1} b_{1}+a_{2} b_{2}$. Then $\langle a, b\rangle$ is a bilinear form on $E_{2}$ and $\langle a, a\rangle=a_{1}^{2}+a_{2}^{2}$ is a quadratic form on $E_{2}$. Put $Q(a)=\langle a, a\rangle$. We consider the field $\Omega$ also as the two-dimensional Euclidean space $E_{2}$ with the scalar product $\langle a, b\rangle$. Then $\|a\|=|a|=\sqrt{Q(a)}, \forall a \in \Omega$.

Proposition 2.2. $\quad$ (i) Equalities $Q(a)=\operatorname{det}\left(P_{a}\right), Q(a b)=Q(a) Q(b),|a b|=$ $|a||b|, Q(a)=\operatorname{det}\left(P_{a}\right)$ hold for all $a, b \in \Omega$.

(ii) Let $a=a_{1}+i a_{2} \in \Omega^{*}$. Then $\operatorname{det}\left(P_{a}\right)=Q(a)=a_{1}^{2}+a_{2}^{2}>0$.

An endomorphism $\psi$ of a vector space $\Omega$ is called an involution of the field $\Omega$ if $\psi(\psi(a))=a$ and $\psi(a b)=\psi(a) \psi(b)$ for all $a, b \in \Omega$. For an element $a=a_{1}+i a_{2} \in \Omega$, we set $\bar{a}=a_{1}-i a_{2}$.

Proposition 2.3. The mapping $a \rightarrow \bar{a}$ is an involution of the field $\Omega$. In addition, for an arbitrary element $a=a_{1}+i a_{2} \in \Omega$, equalities $a+\bar{a}=2 a_{1},<$ $a, a>=a \bar{a}=a_{1}^{2}+a_{2}^{2} \in R$ hold.

Proposition 2.4. Let $a \in \Omega$. Then the element $a^{-1}$ exists if and only if $Q(a) \neq 0$. In the case $Q(a) \neq 0$, equalities $a^{-1}=\frac{\bar{a}}{Q(a)}$ and $Q\left(a^{-1}\right)=\frac{1}{Q(a)}$ hold.

Let $W=\left(\begin{array}{cc}1 & 0 \\ 0 & -1\end{array}\right)$. We will use $W$ also for the writing of the element $\bar{z}$ in the form $\bar{z}=W z$.

Proposition 2.5. $Q(W a)=Q(a)$ for all $a \in \Omega$ and $\langle W a, W b\rangle=\langle a, b\rangle$ for all $a, b \in \Omega$.

Let $a=a_{1}+i a_{2} \in \Omega^{*}$ that is $|a| \neq 0$. Put

$$
P_{a}^{+}=\left(\begin{array}{ll}
\frac{a_{1}}{|a|} & \frac{-a_{2}}{|a|} \\
\frac{a_{2}}{|a|} & \frac{a_{1}}{|a|}
\end{array}\right) .
$$

Proposition 2.6. Let $a=a_{1}+i a_{2} \in \Omega^{*}$. Then the equality $P_{a}=|a| P_{a}^{+}$ holds, where $P_{a}^{+} \in O^{+}(2)$.

Proof. The equality $P_{a}=|a| P_{a}^{+}$is obvious. Since $\left(\frac{a_{1}}{|a|}\right)^{2}+\left(\frac{a_{2}}{|a|}\right)^{2}=1$, the implication $P_{a}^{+} \in O^{+}(2)$ follows from [5, p.161-162].

Put $\Omega^{*}=\{z \in \Omega \mid Q(z) \neq 0\} . \Omega^{*}$ is a group with respect to the multiplication operation in the field $\Omega$. Put $S\left(\Omega^{*}\right)=\{z \in \Omega \mid Q(z)=1\}, P\left(\Omega^{*}\right)=$ $\left\{P_{z} \mid z \in \Omega^{*}\right\}$ and $P\left(S\left(\Omega^{*}\right)\right)=\left\{P_{z} \mid z \in S\left(\Omega^{*}\right)\right\}$. $S\left(\Omega^{*}\right)$ is a subgroup of the 
group $\Omega^{*}$ and $S\left(\Omega^{*}\right)=\left\{e^{i \varphi} \mid \varphi \in R\right\}$. Denote by $P\left(\Omega^{*}\right) W$ the set of all matrices $\left\{g W \mid g \in P\left(\Omega^{*}\right)\right\}$, where $g W$ is the multiplication of matrices $g$ and $W$.

We note that $O^{+}(2) \cap O^{-}(2)=\emptyset$ and the set $O^{+}(2)$ is a subgroup of $O(2)$.

Theorem 2.7. The following equalities are hold:

(i) $O^{+}(2)=\left\{F: E_{2} \rightarrow E_{2} \mid F(x)=P_{a} x, a \in S\left(\Omega^{*}\right), \forall x \in E_{2}\right\}$

(ii) $O^{-}(2)=\left\{F: E_{2} \rightarrow E_{2} \mid F(x)=P_{a} W(x), a \in S\left(\Omega^{*}\right), \forall x \in E_{2}\right\}$

(iii) $O(2)=O^{+}(2) \cup O^{-}(2)$.

Remark 2.8. It is obvious that a $T$-path $x(t)$ in $E_{2}$ is $L$-regular if and only if $Q(x(t)) \neq 0$ for all $t \in T$.

Let $x(t)=\left(x_{1}(t), x_{2}(t)\right)$ be a $T$-path in $E_{2}$ and $x^{\prime}(t)=\left(x_{1}^{\prime}(t), x_{2}^{\prime}(t)\right)$ is its first derivative. Denote the determinant of vectors $x(t), x^{\prime}(t)$ by $\left[x(t) x^{\prime}(t)\right]$.

The following proposition is known.

Proposition 2.9. $\quad$ (i) The functions $Q(x(t))=\langle x(t), x(t)\rangle,\left\langle x(t), x^{\prime}(t)\right\rangle$ and $<x^{\prime}(t), x^{\prime}(t)>$ are $O(2)$-invariant on the set of all $L$-regular $T$-paths $x(t)$ in $E_{2}$;

(ii) The function $\frac{\left\langle x(t), x^{\prime}(t)\right\rangle}{Q(x(t))}$ is $O(2)$-invariant on the set of all L-regular T-paths $x(t)$ in $E_{2}$;

(iii) The function $\left[x(t) x^{\prime}(t)\right]$ is $O^{+}(2)$-invariant on the set of all L-regular T-paths $x(t)$ in $E_{2}$;

(iV) The function $\frac{\left[x(t) x^{\prime}(t)\right]}{Q(x(t))}$ is $O^{+}(2)$-invariant on the set of all L-regular Tpaths $x(t)$ in $E_{2}$;

(V) The function $\frac{\left[x(t) x^{\prime}(t)\right]^{2}}{Q^{2}(x(t))}$ is $O(2)$-invariant on the set of all L-regular $T$ paths $x(t)$ in $E_{2}$;

(Vi) The function $\frac{\left\langle x^{\prime}(t), x^{\prime}(t)\right\rangle}{Q(x(t))}$ is $O(2)$-invariant on the set of all L-regular T-paths $x(t)$ in $E_{2}$. 


\section{Complete systems of invariants of an L-regular path and uniqueness theorems for $L$-regular paths}

Proposition 3.1. Let $u, v \in \Omega$. Assume that $Q(u) \neq 0$. Then the element $v u^{-1}$ exists, the following equalities hold:

$$
v u^{-1}=\frac{<u, v>}{Q(u)}+i \frac{[u v]}{Q(u)}
$$

and

$$
P_{v u^{-1}}=\left(\begin{array}{cc}
\frac{\langle u, v>}{Q(u)} & -\frac{[u v]}{Q(u)} \\
\frac{[u v]}{Q(u)} & \frac{<u, v>}{Q(u)}
\end{array}\right) .
$$

Proof. Let $u=u_{1}+i u_{2}, v=v_{1}+i v_{2}$. Since $Q(u) \neq 0$, element $u^{-1}$ exists by Proposition 2.4. Hence $v u^{-1}$ exists. By Proposition 2.4, $u^{-1}=\frac{\bar{u}}{Q(u)}$. Using $\bar{u}=u_{1}-i u_{2}$ and the multiplication in the field $\Omega$, we obtain the equality $v u^{-1}=\frac{\langle u, v>}{Q(u)}+i \frac{[u v]}{Q(u)}$. This equality and the notation of $P_{a}$ imply (2).

Theorem 3.2. $\quad$ (i) Let $x(t)$ and $y(t)$ be L-regular T-paths in $E_{2}$ such that $x(t) \stackrel{O^{+}(2)}{\sim} y(t)$. Then, for all $t \in T$, the following equalities hold

$$
\left\{\begin{aligned}
<x(t), x(t)> & =<y(t), y(t)>, \\
{\left[x(t) x^{\prime}(t)\right] } & =\left[y(t) y^{\prime}(t)\right] .
\end{aligned}\right.
$$

(ii) Conversely, if $x(t)$ and $y(t)$ are $L$-regular T-paths in $E_{2}$ such that the equalities (3) hold, then $x(t) \stackrel{O^{+}(2)}{\sim} y(t)$. Moreover, there exists the unique $F \in O^{+}(2)$ such that $y(t)=F x(t)$ for all $t \in T$. In this case, $F=U$, where $U \in O^{+}(2), U$ has the following form

$$
U=\left(\begin{array}{cc}
\frac{<x(t), y(t)>}{Q(x(t))} & -\frac{[x(t) y(t)]}{Q(x(t))} \\
\frac{[x(t) y(t)]}{Q(x(t))} & \frac{<x(t), y(t)>}{Q(x(t))}
\end{array}\right),
$$

and the matrix $U$ does not depend on $t \in T$.

Proof. (i) Assume that $x(t) \stackrel{O^{+}(2)}{\sim} y(t)$. Then $g \in O^{+}(2)$ exists such that $y(t)=g x(t)$ for all $t \in T$. Using this equality, we obtain $\langle y(t), y(t)\rangle=<$ $g x(t), g x(t)>=<x(t), x(t)>$ for all $t \in T$. Hence the equality $<$ $x(t), x(t)>=<y(t), y(t)>$ holds for all $t \in T$. Using $\operatorname{det}(g)=1$, we obtain $\left[y(t) y^{\prime}(t)\right]=\left[g x(t) g x^{\prime}(t)\right]=\operatorname{det}(g)\left[x(t) x^{\prime}(t)\right]=\left[x(t) x^{\prime}(t)\right]$. Hence the equality $\left[x(t) x^{\prime}(t)\right]=\left[y(t) y^{\prime}(t)\right]$ holds for all $t \in T$. 
(ii) Conversely, assume that equalities (3) hold. Since $x(t)$ and $y(t)$ are $L$ regular paths, we have $Q(x(t)) \neq 0$ and $Q(y(t)) \neq 0$ for all $t \in T$. Then, by Proposition 2.4, $(x(t))^{-1}$ and $(y(t))^{-1}$ exist for all $t \in T$. We put $u=y(t), v=y^{\prime}(t)$ in Proposition 3.1. Then we obtain

$$
\frac{y^{\prime}(t)}{y(t)}=\frac{<y(t), y^{\prime}(t)>+i\left[y(t) y^{\prime}(t)\right]}{Q(y(t))} .
$$

Similarly, we obtain

$$
\frac{x^{\prime}(t)}{x(t)}=\frac{<x(t), x^{\prime}(t)>+i\left[x(t) x^{\prime}(t)\right]}{Q(x(t))} .
$$

Equalities $<x(t), x^{\prime}(t)>=\frac{1}{2} \frac{d}{d t}<x(t), x(t)>,<y(t), y^{\prime}(t)>=\frac{1}{2} \frac{d}{d t}<$ $y(t), y(t)>$ and the equality $\langle x(t), x(t)>=<y(t), y(t)>$ in the equalities (3) imply the equality $\left\langle x(t), x^{\prime}(t)>=<y(t), y^{\prime}(t)>\right.$ for all $t \in T$. This equality, the equality $Q(x(t))=<x(t), x(t)>=<y(t), y(t)>=$ $Q(y(t))$, the equality $\left[x(t) x^{\prime}(t)\right]=\left[y(t) y^{\prime}(t)\right]$ in $(3)$ and the equalities (5), (6) imply the equality $y^{\prime}(t)(y(t))^{-1}=x^{\prime}(t)(x(t))^{-1}$. Hence $y^{\prime}(t)(y(t))^{-1}-x^{\prime}(t)(x(t))^{-1}=0$ for all $t \in T$. Using this equality, we obtain

$$
\begin{array}{r}
\frac{d\left(y(t)(x(t))^{-1}\right)}{d t}=y^{\prime}(t)(x(t))^{-1}-y(t) x^{\prime}(t)(x(t))^{-2}= \\
y(t)\left(y^{\prime}(t)(y(t))^{-1}-x^{\prime}(t)(x(t))^{-1}\right)(x(t))^{-1}=0
\end{array}
$$

for all $t \in T$. This means that the function $y(t)(x(t))^{-1}$ is a constant on $T$. Put $g=y(t)(x(t))^{-1}$. Since $Q(x(t)) \neq 0$ and $Q(y(t)) \neq 0$ for all $t \in T$, we have $g \neq 0$.

Since $\left.y(t)=y(t)(x(t))^{-1} x(t)\right)=\left(y(t)(x(t))^{-1}\right) x(t)$, we have $y(t)=$ $g x(t)$. Using (1), we obtain $y(t)=g x(t)=P_{g} x(t)$. The equality $<x(t), x(t)>=<y(t), y(t)>$ in $(3)$ implies

$<y(t), y(t)>=<P_{g} x(t), P_{g} x(t)>=<x(t), x(t)>=$ for all $t \in T$. Since $P_{g}$ is linear, we obtain $P_{g} \in O(2)$. Then $\operatorname{det}\left(P_{g}\right)=1$ or $\operatorname{det}\left(P_{g}\right)=-1$. Using the equality $\left[x(t) x^{\prime}(t)\right]=\left[y(t) y^{\prime}(t)\right]$ in the equalities $(3)$, we obtain that $\operatorname{det}\left(P_{g}\right)=1$ and $P_{g} \in O^{+}(2)$. Hence $x(t) \stackrel{O^{+}(2)}{\sim} y(t)$.

By $g=y(t)(x(t))^{-1}=\frac{<x(t), y(t)>}{Q(x(t))}+i \frac{[x(t) y(t)]}{Q(x(t))}$ and Proposition 3.1, $P_{g}$ has the form (4) and $P_{g}=U$. Since $g$ is a constant, the matrix $U$ does not depend on $t \in T$.

Prove the uniqueness of $U \in O^{+}(2)$ satisfying the condition $y(t)=U x(t)$. Assume that $H \in O^{+}(2)$ exists such that $y(t)=H x(t)$. Then by $(1)$, 
Proposition 2.1 and Theorem 2.7 $(i)$, there exists the unique $a \in \Omega^{*}$ such that $H=P_{a}$. Hence we have $y(t)=P_{a} x(t)$. By $(1)$, we obtain $y(t)=$ $a x(t)$. Since $Q(x(t)) \neq 0, y(t)=a x(t)$ implies that $a=y(t)(x(t))^{-1}=g$. Hence $P_{a}=P_{g}=U$. The uniqueness of $U$ is proved.

Example 3.3. Consider $L$-regular $T$-paths $x(t)=\left(\frac{t^{4}+1}{t^{2}+1}, \frac{t^{2}}{t^{2}+1}\right)$ and $y(t)=\left(\frac{\sqrt{3}-t^{2}+\sqrt{3} t^{4}}{2+2 t^{2}}, \frac{1+\sqrt{3} t^{2}+t^{4}}{2+2 t^{2}}\right)$, where $T=R$. It is easy to see that equalities in the equalities (3) hold for these paths $x(t)$ and $y(t)$. Then, by Theorem $3.2(i i), x(t) \stackrel{O^{+}(2)}{\sim} y(t)$. Moreover, by Theorem 3.2(ii), we obtain that $U=$ $\left(\begin{array}{cc}\sqrt{3} / 2 & -1 / 2 \\ 1 / 2 & \sqrt{3} / 2\end{array}\right)$

Definition 3.4. A $T$-path $x(t)$ in $E_{2}$ is called completely $L$-degenerate if $\left[x(t) x^{\prime}(t)\right]=0$ for all $t \in T$.

A $T$-path $x(t)$ in $E_{2}$ is called L-non-degenerate if $\left[x(t) x^{\prime}(t)\right] \neq 0$ for all $t \in T$.

It is obvious that every L-non-degenerate path is L-regular. If $x(t)$ and $y(t)$ are $T$-paths in $E_{2}$ such that $x(t)$ is completely L-degenerate and $x \stackrel{O(2)}{\sim} y$, then $y(t)$ is also completely L-degenerate. Similarly, it is obvious that if $x(t)$ is L-non-degenerate and $x \stackrel{O(2)}{\sim} y$, then $y(t)$ is also L-non-degenerate.

Example 3.5. Consider the $T$-path $x(t)=\left(t^{2}, \frac{t^{2}}{2}\right)$ in $E_{2}$, where $T=(0,1)$. Then, $\left[x(t) x^{\prime}(t)\right]=0$ for all $t \in T$. That is, $x(t)$ is a completely $L$-degenerate $T$-path. Since $t^{2} \neq 0$ for all $t \in T$, we obtain that $x(t)=\left(t^{2}, \frac{t^{2}}{2}\right)$ is a completely $L$-degenerate $L$-regular $T$-path.

Theorem 3.6. (i) Let $x(t), y(t)$ be completely L-degenerate L-regular $T$ paths in $E_{2}$ such that $x(t) \stackrel{O(2)}{\sim} y(t)$. Then, for all $t \in T$, the following equality holds:

$$
<x(t), x(t)>=<y(t), y(t)>.
$$

(ii) Conversely, if $x(t)$ and $y(t)$ are completely L-degenerate L-regular $T$ paths in $E_{2}$ such that the equality $(7)$ holds, then $x(t) \stackrel{O(2)}{\sim} y(t)$. Moreover, only two elements $F=F_{1}, F_{2} \in O(2)$ exist such that $y(t)=F(x(t))$ for all $t \in T$. Here $F_{1}=U_{1}, F_{2}=\left(U_{2} W\right)$, where $U_{1}, U_{2} \in O^{+}(2)$, the matrix $U_{1}$ has the form (4) and the matrix $U_{2}$ has the form

$$
U_{2}=\left(\begin{array}{cc}
\frac{\leq W x(t), y(t)>}{Q(W x(t))} & -\frac{[W x(t) y(t)]}{Q(W x(t))} \\
\frac{[W x(t) y(t)]}{Q(W x(t))} & \frac{\leq W x(t), y(t)>}{Q(W x(t))}
\end{array}\right) .
$$

The matrices $U_{1}$ and $U_{2}$ are independent from variable on $t \in T$. 
Proof. (i) Assume that $x(t) \stackrel{O(2)}{\sim} y(t)$. The function $\langle x(t), x(t)\rangle$ is $O(2)$ invariant, so the equality (7) holds.

(ii) Conversely, assume that equality (7) holds. Since $x(t)$ and $y(t)$ are completely L-degenerate paths, we have

$$
\left[x(t) x^{\prime}(t)\right]=\left[y(t) y^{\prime}(t)\right]=0 .
$$

for all $t \in T$. This equality and the equality (7) imply the equalities (3). Then, by Theorem 3.2(ii), the unique $F \in O^{+}(2)$ exists such that $y(t)=F x(t)$ for all $t \in T$. This means that the unique $U_{1} \in O^{+}(2)$ exists such that $y(t)=U_{1} x(t)$ for all $t \in T$. Here, the matrix $U_{1}$ has the form (4). By Theorem 3.2, the matrix $U_{1}$ does not depend on $t \in T$.

Consider the $T$-path $W x(t)$. Since the function $\langle x(t), x(t)\rangle$ is $W$ invariant, we have $<W x(t), W x(t)>=<x(t), x(t)>$ for all $t \in T$. Similarly we obtain $<W y(t), W y(t)>=<y(t), y(t)>$ for all $t \in T$. Using $\operatorname{det}(W)=-1$ and the equality (9) for all $t \in T$, we obtain

$\left[W x(t)(W x)^{\prime}(t)\right]=\operatorname{det}(W)\left[x(t) x^{\prime}(t)\right]=-\left[x(t) x^{\prime}(t)\right]=\left[y(t) y^{\prime}(t)\right]=0$

Using the equalities $<W x(t), W x(t)>=<x(t), x(t)>$ and $\left.\left[(W x(t))(W x)^{\prime}(t)\right)\right]=\left[y(t) y^{\prime}(t)\right]=0$, we obtain equalities:

$$
\left\{\begin{array}{l}
<W x(t), W x(t)>=<y(t), y(t)> \\
{\left[W x(t)(W x)^{\prime}(t)\right]=\left[y(t) y^{\prime}(t)\right]}
\end{array}\right.
$$

for all $t \in T$. Then, by Theorem 3.2(ii), the unique $U_{2} \in O^{+}(2)$ exists such that $y(t)=U_{2}(W x(t))=\left(U_{2} W\right) x(t)$ for all $t \in T$. Here, the matrix $U_{2}$ has the form (8). By Theorem 3.2, the matrix $U_{2}$ does not depend on $t \in T$.

Let $F \in O(2)$ such that $y(t)=F x(t)$. Prove that $F x(t)=U_{1} x(t)$ or $F x(t)=\left(U_{2} W\right) x(t)$. Let $y(t)=F x(t)=C x$ for some $C \in O(2)$. Then $C \in O^{+}(2)$ or $C \in O^{-}(2)=O^{+}(2) W$. Assume that $C \in O^{+}(2)$. Then, by the uniqueness in Theorem 3.2, $C=U_{1}$. Assume that $C \in$ $O^{+}(2) W$. Then $C$ has the form $C=D W$, where $D \in O^{+}(2)$. We have $y(t)=(D W) x(t)=D(W x(t))$. Hence paths $y(t)$ and $W x(t)$ are $O^{+}(2)$-equivalent. By the uniqueness in Theorem 3.2, $D=U_{2}$.

Theorem 3.7. (i) Let $x(t)$ and $y(t)$ be L-non-degenerate T-paths in $E_{2}$ such that $x \stackrel{O(2)}{\sim} y$. Then, for all $t \in T$, the following equalities hold 


$$
\left\{\begin{array}{c}
<x(t), x(t)>=<y(t), y(t)>, \\
{\left[x(t) x^{\prime}(t)\right]^{2}=\left[y(t) y^{\prime}(t)\right]^{2} .}
\end{array}\right.
$$

(ii) Conversely, if $x(t)$ and $y(t)$ are $L$-non-degenerate $T$-paths in $E_{2}$ such that the equalities (10) hold, then $x \stackrel{O(2)}{\sim} y$. Moreover, the unique $F \in O(2)$ exists such that $y(t)=F x(t)$ for all $t \in T$. In this case, the following cases exist:

(a) $\left[x(t) x^{\prime}(t)\right]>0$ and $\left[y(t) y^{\prime}(t)\right]>0$ for all $t \in T$;

(b) $\left[x(t) x^{\prime}(t)\right]>0$ and $\left[y(t) y^{\prime}(t)\right]<0$ for all $t \in T$;

(c) $\left[x(t) x^{\prime}(t)\right]<0$ and $\left[y(t) y^{\prime}(t)\right]>0$ for all $t \in T$;

(d) $\left[x(t) x^{\prime}(t)\right]<0$ and $\left[y(t) y^{\prime}(t)\right]<0$ for all $t \in T$.

In cases $(a)$ and $(d), F=U_{1}$, where $U_{1} \in O^{+}(2)$ and the matrix $U_{1}$ has the form (4).

In cases $(b)$ and $(c), F=\left(U_{2} W\right)$, where $U_{2} \in O^{+}(2)$ and the matrix $U_{2}$ has the form (8).

Proof. (i) Let $x \stackrel{O(2)}{\sim} y$. The functions $<x(t), x(t)>$ and $\left[x(t) x^{\prime}(t)\right]^{2}$ are $O(2)$-invariant for all $t \in T$. So equalities (10) hold.

(ii) Conversely, assume that equalities (10) hold. Since $x(t)$ and $y(t)$ are $L$ non-degenerate $T$-paths, $\left[x(t) x^{\prime}(t)\right] \neq 0$ and $\left[y(t) y^{\prime}(t)\right] \neq 0$ for all $t \in T$. Hence there exist the following cases:

(a) $\left[x(t) x^{\prime}(t)\right]>0$ and $\left[y(t) y^{\prime}(t)\right]>0$ for all $t \in T$;

(b) $\left[x(t) x^{\prime}(t)\right]>0$ and $\left[y(t) y^{\prime}(t)\right]<0$ for all $t \in T$;

(c) $\left[x(t) x^{\prime}(t)\right]<0$ and $\left[y(t) y^{\prime}(t)\right]>0$ for all $t \in T$; 
(d) $\left[x(t) x^{\prime}(t)\right]<0$ and $\left[y(t) y^{\prime}(t)\right]<0$ for all $t \in T$.

By the equalities (10), we have the equality $\left[x(t) x^{\prime}(t)\right]^{2}=\left[y(t) y^{\prime}(t)\right]^{2}$. In cases $(a)$ and $(d)$ this equality implies $\left[x(t) x^{\prime}(t)\right]=\left[y(t) y^{\prime}(t)\right]$. Then, by Theorem 3.2, we obtain that there exists the unique $F \in O^{+}(2)$ such that $y(t)=F x(t)$ for all $t \in T$ and $F x(t)=U_{1} x(t)$, where the matrix $U_{1}$ has the form (4).

In cases $(b)$ and $(c)$, the equality $\left[x(t) x^{\prime}(t)\right]^{2}=\left[y(t) y^{\prime}(t)\right]^{2}$ implies $\left[x(t) x^{\prime}(t)\right]=-\left[y(t) y^{\prime}(t)\right]$ for all $t \in T$. Using this equality and $\operatorname{det}(W)=-1$, we obtain

$$
\left[W x(t)(W x)^{\prime}(t)\right]=\operatorname{det}(W)\left[x(t) x^{\prime}(t)\right]=-\left[x(t) x^{\prime}(t)\right]=\left[y(t) y^{\prime}(t)\right]
$$

for all $t \in T$. This equality and the equality $<W x(t), W x(t))=<x(t), x(t)>=<y(t), y(t)>$ imply the equalities (3) for paths $W x(t)$ and $y(t)$. By Theorem 3.2(ii), the unique $U_{2} \in O^{+}(2)$ exists such that $y(t)=U_{2}(W x(t))$, where $U_{2}$ has the form (8). Since $U_{2} \in O^{+}(2)$, we have $U_{2} W \in O^{+}(2) W$.

Lemma 3.8. For all vectors $y_{1}, y_{2}, z_{1}, z_{2}$ in $E_{2}$, the equality $\left[y_{1} y_{2}\right]\left[z_{1} z_{2}\right]=<$ $y_{1}, z_{1}><y_{2}, z_{2}>-<y_{1}, z_{2}><y_{2}, z_{1}>$ holds.

Proof. A proof of the this lemma is given in ([16, Lemma 13]).

Theorem 3.9. $\quad$ (i) Let $x(t), y(t)$ be L-non-degenerate T-paths in $E_{2}$ such that $x \stackrel{O(2)}{\sim} y$. Then, for all $t \in T$, the following equalities hold

$$
\left\{\begin{array}{c}
<x(t), x(t)>=<y(t), y(t)>, \\
<x^{\prime}(t), x^{\prime}(t)>=<y^{\prime}(t), y^{\prime}(t)>.
\end{array}\right.
$$

(ii) Conversely, if $x(t)$ and $y(t)$ are L-non-degenerate T-paths in $E_{2}$ such that the equalities (11) hold, then $x \stackrel{O(2)}{\sim} y$. Moreover, the unique $F \in O(2)$ exists such that $y(t)=F x(t)$ for all $t \in T$. In this case, only cases $(a),(b),(c),(d)$ in Theorem 3.7 exist.

In cases $(a)$ and $(d), F=U_{1}$, where $U_{1} \in O^{+}(2)$ and $U_{1}$ has the form (4).

In cases (b) and $(c), F=\left(U_{2} W\right)$, where $U_{2} \in O^{+}(2)$ and $U_{2}$ has the form (8).

Proof. (i) Let $x \stackrel{O(2)}{\sim} y$. Since functions $\langle x(t), x(t)\rangle$ and $\left\langle x^{\prime}(t), x^{\prime}(t)\right\rangle$ are $O(2)$-invariants, so the equalities (11) hold. 
(ii) Conversely, assume that the equalities (11) hold.

Applying Lemma 3.8 to vectors $y_{1}=z_{1}=x(t), y_{2}=z_{2}=x^{\prime}(t)$, we obtain the following equality

$$
\left[x(t) x^{\prime}(t)\right]^{2}=<x(t), x(t)><x^{\prime}(t), x^{\prime}(t)>-<x(t), x^{\prime}(t)>^{2}
$$

for all $t \in T$.

As in the proof of Theorem 3.2, the equality $\langle x(t), x(t)>=<y(t), y(t)>$ for all $t \in T$ implies the equality $\left\langle x(t), x^{\prime}(t)\right\rangle=<y(t), y^{\prime}(t)>$ for all $t \in T$. Using the equalities (11), the equality $<x(t), x^{\prime}(t)>=<y(t), y^{\prime}(t)>$ and the equality (12) for all $t \in T$, we obtain the equalities (10). Then the proof of the present theorem follows from Theorem 3.7.

\section{Theorems on an existence for L-regular paths}

Theorem 4.1. (i) Let $a(t)$ be a real $C^{(1)}$-function on $T$ such that $a(t)>0$ for all $t \in T$, and $b(t)$ be a real continuous function on $T$. Then there exists an $L$-regular $T$-path $x(t)$ in $E_{2}$ such that

$$
\left\{\begin{aligned}
<x(t), x(t)> & =a(t), \\
{\left[x(t) x^{\prime}(t)\right] } & =b(t)
\end{aligned}\right.
$$

for all $t \in T$. Moreover, a general solution of this system of equations has the following form

$$
x(t)=e^{i \varphi} \sqrt{a(t)} e^{i \int_{t_{0}}^{t} \frac{b(u)}{a(u)} d u},
$$

where $\varphi \in[0,2 \pi)$ and $t_{0} \in T$.

(ii) Conversely, every path of the form (14) in $E_{2}$ is an L-regular path such that the equalities (13) hold.

Proof. (i) Assume that an $L$-regular path $x(t)$ in $E_{2}$ such that the equalities (13) hold. The identity $v u^{-1}=\frac{\langle u, v\rangle}{Q(u)}+i \frac{[u v]}{Q(u)}$ in Proposition 3.1 implies the following identity for the $L$-regular path $x(t)$ :

$$
x^{\prime}(t)(x(t))^{-1}=\frac{<x(t), x^{\prime}(t)>}{Q(x(t))}+i \frac{\left[x(t) x^{\prime}(t)\right]}{Q(x(t))} .
$$

This identity, (13) and the equality $\left\langle x(t), x^{\prime}(t)\right\rangle=\frac{1}{2} \frac{d}{d t}<x(t), x(t)>$ imply the equality:

$$
\frac{x^{\prime}(t)}{x(t)}=\frac{1}{2} \frac{a^{\prime}(t)}{a(t)}+i \frac{b(t)}{a(t)} .
$$


Then we obtain the following differential equation for the path $x(t)$ :

$$
x^{\prime}(t)=\left(\frac{1}{2} \frac{a^{\prime}(t)}{a(t)}+i \frac{b(t)}{a(t)}\right) x(t) .
$$

This differential equation has the following general solution:

$$
x(t)=K e^{\int_{t_{0}}^{t}\left(\frac{1}{2} \frac{a^{\prime}(u)}{a(u)}+i \frac{b(u)}{a(u)}\right) d u},
$$

where $K \in \Omega$. Since $x(t)$ is an $L$-regular path, we have $x(t) \neq 0$ for all $t \in T$. This inequality and the last above equality imply that $K \neq 0$. Using equalities $\langle x(t), x(t)\rangle=x(t) \overline{x(t)}$ and $\langle x(t), x(t)\rangle=a(t)$, we obtain the following equality

$$
\begin{array}{r}
a(t)=<x(t), x(t)>=<K e^{\int_{t_{0}}^{t}\left(\frac{1}{2} \frac{a^{\prime}(u)}{a(u)}+i \frac{b(u)}{a(u)}\right) d u}, K e^{\int_{t_{0}}^{t}\left(\frac{1}{2} \frac{a^{\prime}(u)}{a(u)}+i \frac{b(u)}{a(u)}\right) d u}> \\
=K e^{\int_{t_{0}}^{t}\left(\frac{1}{2} \frac{a^{\prime}(u)}{a(u)}+i \frac{b(u)}{a(u)}\right) d u} \bar{K} e^{\int_{t_{0}}^{t}\left(\frac{1}{2} \frac{a^{\prime}(u)}{a(u)}-i \frac{b(u)}{a(u)}\right) d u} \\
=|K|^{2} e^{\int_{t_{0}}^{t}\left(\frac{1}{2} \frac{a^{\prime}(u)}{a(u)}\right) d u} e^{\int_{t_{0}}^{t}\left(\frac{1}{2} \frac{a^{\prime}(u)}{a(u)}\right) d u} .
\end{array}
$$

This equality implies the equality $|K| e^{\int_{t_{0}}^{t}\left(\frac{1}{2} \frac{a^{\prime}(u)}{a(u)}\right) d u}=\sqrt{a(t)}$. Using this equality and the equality $K=|K| e^{i \varphi}$, where $0 \leq \varphi<2 \pi$, we obtain

$$
\begin{array}{r}
x(t)=K e^{\int_{t_{0}}^{t}\left(\frac{1}{2} \frac{a^{\prime}(u)}{a(u)}+i \frac{b(u)}{a(u)}\right) d u}=|K| e^{\int_{t_{0}}^{t}\left(\frac{1}{2} \frac{a^{\prime}(u)}{a(u)} d u\right.} e^{i \varphi} e^{\int_{t_{0}}^{t}\left(i \frac{b(u)}{a(u)}\right) d u} \\
=e^{i \varphi} \sqrt{a(t)} e^{\int_{t_{0}}^{t}\left(i \frac{b(u)}{a(u)}\right) d u .} .
\end{array}
$$

This equality implies the form (14).

(ii) Conversely, assume that an $L$-regular path $x(t)$ has the form (14). Then, we have $x(t)=e^{i \varphi} \sqrt{a(t)} e^{i \int_{t_{0}}^{t} \frac{b(u)}{a(u)} d u}$. This equality implies

$$
\begin{aligned}
<x(t), x(t)> & =<e^{i \varphi} \sqrt{a(t)} e^{i \int_{t_{0}}^{t} \frac{b(u)}{a(u)} d u}, e^{i \varphi} \sqrt{a(t)} e^{i \int_{t_{0}}^{t} \frac{b(u)}{a(u)} d u}>= \\
& e^{i \varphi} \sqrt{a(t)} e^{i \int_{t_{0}}^{t} \frac{b(u)}{a(u)} d u} e^{-i \varphi} \sqrt{a(t)} e^{-i \int_{t_{0}}^{t} \frac{b(u)}{a(u)} d u}=a(t) .
\end{aligned}
$$

Hence $\left\langle x(t), x(t)>=a(t)\right.$. The equality $x(t)=e^{i \varphi} \sqrt{a(t)} e^{i \int_{t_{0}}^{t} \frac{b(u)}{a(u)} d u}$ implies

$$
x^{\prime}(t)=e^{i \varphi} \frac{1}{2} \frac{a^{\prime}(t)}{\sqrt{a(t)}} e^{i \int_{t_{0}}^{t} \frac{b(u)}{a(u)} d u}+e^{i \varphi} \sqrt{a(t)} e^{i \int_{t_{0}}^{t} \frac{b(u)}{a(u)} d u}\left(i \frac{b(t)}{a(t)}\right) .
$$


This equality and the equality $x(t)=e^{i \varphi} \sqrt{a(t)} e^{i \int_{t_{0}}^{t} \frac{b(u)}{a(u)} d u}$ imply $\left[x(t) x^{\prime}(t)\right]=$

$$
\begin{aligned}
& {\left[e^{i \varphi} \sqrt{a(t)} e^{i \int_{t_{0}}^{t} \frac{b(u)}{a(u)} d u} \quad\left(e^{i \varphi} \frac{1}{2} \frac{a^{\prime}(t)}{\sqrt{a(t)}} e^{i \int_{t_{0}}^{t} \frac{b(u)}{a(u)} d u}+e^{i \varphi} \sqrt{a(t)} e^{i \int_{t_{0}}^{t} \frac{b(u)}{a(u)} d u}\left(i \frac{b(t)}{a(t)}\right)\right)\right]=} \\
& {\left[e^{i \varphi} \sqrt{a(t)} e^{i \int_{t_{0}}^{t} \frac{b(u)}{a(u)} d u} \quad e^{i \varphi} \frac{1}{2} \frac{a^{\prime}(t)}{\sqrt{a(t)}} e^{i \int_{t_{0}}^{t} \frac{b(u)}{a(u)} d u}\right]+} \\
& {\left[e^{i \varphi} \sqrt{a(t)} e^{i \int_{t_{0}}^{t} \frac{b(u)}{a(u)} d u} e^{i \varphi} \sqrt{a(t)} e^{i \int_{t_{0}}^{t} \frac{b(u)}{a(u)} d u}\left(i \frac{b(t)}{a(t)}\right)\right]=} \\
& \frac{1}{2} a^{\prime}(t)\left[e^{i \varphi} e^{i \int_{t_{0}}^{t} \frac{b(u)}{a(u)} d u} e^{i \varphi} e^{i \int_{t_{0}}^{t} \frac{b(u)}{a(u)} d u}\right]+b(t)\left[e^{i \varphi} e^{i \int_{t_{0}}^{t} \frac{b(u)}{a(u)} d u} \quad i e^{i \varphi} e^{i \int_{t_{0}}^{t} \frac{b(u)}{a(u)} d u}\right]=
\end{aligned}
$$

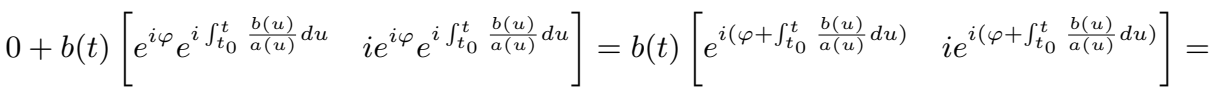

$$
\begin{aligned}
& b(t)\left|\begin{array}{cc}
\cos \left(\varphi+\int_{t_{0}}^{t} \frac{b(u)}{a(u)} d u\right) & \sin \left(\varphi+\int_{t_{0}}^{t} \frac{b(u)}{a(u)} d u\right) \\
-\sin \left(\varphi+\int_{t_{0}}^{t} \frac{b(u)}{a(u)} d u\right) & \cos \left(\varphi+\int_{t_{0}}^{t} \frac{b(u)}{a(u)} d u\right)
\end{array}\right|=b(t) .
\end{aligned}
$$

Hence $\left[x(t) x^{\prime}(t)\right]=b(t)$ and the equalities (13) hold.

Example 4.2. Let $T=R$. Consider the following real continuous functions on $T: a(t)=\frac{4}{1+4 t^{2}}$ and $b(t)=\frac{-8}{\left(1+4 t^{2}\right)^{2}}$. In this case, the system of the equalities (13) has the following general solution:

$$
\begin{aligned}
x(t)=e^{i \varphi} \sqrt{a(t)} e^{i \int_{t_{0}}^{t} \frac{b(u)}{a(u)} d u} & =e^{i \varphi} \frac{2}{\sqrt{1+4 t^{2}}} e^{i \int_{0}^{t} \frac{-2}{1+4 u^{2}} d u} \\
& =e^{i \varphi} \frac{2}{\sqrt{1+4 t^{2}}} e^{-i \arctan (2 t)} \\
& =e^{i \varphi} \frac{2}{\sqrt{1+4 t^{2}}}\left(\frac{1}{\sqrt{1+4 t^{2}}}-i \frac{2 t}{\sqrt{1+4 t^{2}}}\right) \\
& =e^{i \varphi}\left(\frac{2}{1+4 t^{2}}-i \frac{4 t}{1+4 t^{2}}\right)
\end{aligned}
$$

where $\varphi \in[0,2 \pi)$.

Theorem 4.3. (i) Let $a(t)$ be a real $C^{(1)}$-function on $T$ such that $a(t)>0$ for all $t \in T$. Then there exists a completely $L$-degenerate $L$-regular T-path $x(t)$ in $E_{2}$ such that

$$
<x(t), x(t)>=a(t)
$$

for all $t \in T$. Moreover, a general solution of this equation has the following form

$$
x(t)=e^{i \varphi} \sqrt{a(t)},
$$


where $\varphi \in[0,2 \pi)$.

(ii) Conversely, every T-path $x(t)$ in $E_{2}$ of the form (15) is a completely $L$-degenerate $L$-regular path.

Proof. (i) Assume that $x(t)$ is a completely $L$-degenerate $L$-regular $T$-path in $E_{2}$ such that $\langle x(t), x(t)\rangle=a(t)$. Since $x(t)$ is completely $L$ degenerate, we have $\left[x(t) x^{\prime}(t)\right]=0$ for all $t \in T$. Then, using Theorem 4.1 to $x(t)$, we obtain the form (15).

(ii) Conversely, assume that $x(t)$ has the form (15). We have $x(t)=e^{i \varphi} \sqrt{a(t)}$ and $x^{\prime}(t)=\frac{1}{2} e^{i \varphi} \frac{a^{\prime}(t)}{\sqrt{a(t)}}$. Then we obtain

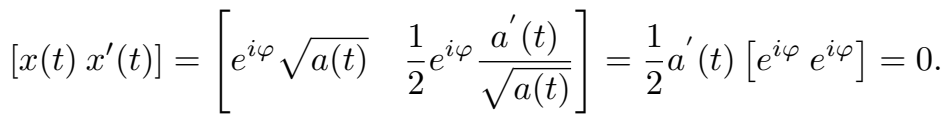

Hence the path (15) is completely $L$-degenerate. It is obvious that the path (15) is $L$-regular.

Theorem 4.4. (i) For every L-non-degenerate T-path $x(t)$ in $E_{2}$, conditions $Q(x)>0$ and $\left[x(t) x^{\prime}(t)\right]^{2}>0$ hold for all $t \in T$.

(ii) Let $t_{0} \in T, a(t)$ be a real $C^{(1)}$-function on $T$ such that $a(t)>0$ for all $t \in T$, and $c(t)$ be a real continuous function on $T$ such that $c(t)>0$ for all $t \in T$. Then there exists an L-non-degenerate $T$-path $x(t)$ in $E_{2}$ such that

$$
\left\{\begin{array}{c}
<x(t), x(t)>=a(t) \\
{\left[x(t) x^{\prime}(t)\right]^{2}=c(t)}
\end{array}\right.
$$

for all $t \in T$. Moreover, a general solution of this system of equations has the following form

$$
x(t)=e^{i \varphi} \sqrt{a(t)} e^{i \int_{t_{0}}^{t} \frac{\sqrt{c(u)}}{a(u)} d u}
$$

or the following form

$$
x(t)=e^{i \varphi} \sqrt{a(t)} e^{-i \int_{t_{0}}^{t} \frac{\sqrt{c(u)}}{a(u)} d u},
$$

where $\varphi \in[0,2 \pi)$. Conversely, $T$-paths of forms (16) and (17) are L-nondegenerate $T$-paths in $E_{2}$ such that $Q(x)=a(t)$ and $\left[x^{\prime}(t) x^{(2)}(t)\right]^{2}=c(t)$. 
Proof. (i) It is obvious.

(ii) Assume that $x(t)$ is an $L$-non-degenerate $T$-path in $E_{2}$ such that $Q(x)=$ $a(t)$ and $\left[x(t) x^{\prime}(t)\right]^{2}=c(t)$. Prove that $x(t)$ has the form (16) or (17). Using $c(t)>0$ for all $t \in T$, we obtain $\left[x(t) x^{\prime}(t)\right]^{2}=(\sqrt{c(t)})^{2}$. This equality implies $\left(\left[x(t) x^{\prime}(t)\right]+\sqrt{c(t)}\right)\left(\left[x(t) x^{\prime}(t)\right]-\sqrt{c(t)}\right)=0$ for all $t \in T$. Since $x(t)$ is an $L$-non-degenerate $T$-path, there exists only following two cases:

(ii.1) $\left[x(t) x^{\prime}(t)\right]>0$ for all $t \in T$

(ii.2) $\left[x(t) x^{\prime}(t)\right]<0$ for all $t \in T$.

Since $\sqrt{c(t)})>0$ for all $t \in T$, in the case $(i i .1)$, the equality $\left(\left[x(t) x^{\prime}(t)\right]+\right.$ $\sqrt{c(t)})\left(\left[x(t) x^{\prime}(t)\right]-\sqrt{c(t)}\right)=0$ implies $\left[x(t) x^{\prime}(t)\right]-\sqrt{c(t)}=0$ for all $t \in T$. Since $\sqrt{c(t)})>0$ for all $t \in T$, in the case (ii.2), the equality $\left(\left[x(t) x^{\prime}(t)\right]+\sqrt{c(t)}\right)\left(\left[x(t) x^{\prime}(t)\right]-\sqrt{c(t)}\right)=0$ implies $\left[x(t) x^{\prime}(t)\right]+\sqrt{c(t)}=$ 0 for all $t \in T$. Consider the following two cases:

(j) $\left[x(t) x^{\prime}(t)\right]-\sqrt{c(t)}=0$ for all $t \in T$;

(jj) $\left[x(t) x^{\prime}(t)\right]+\sqrt{c(t)}=0$ for all $t \in T$.

In the case $(j)$, we have $\left[x(t) x^{\prime}(t)\right]=\sqrt{c(t)}$ and $Q(x)=a(t)$. Then, using Theorem 4.1, where $b(t)=\sqrt{c(t)})$, we obtain the path (16). As in Theorem 4.1, it is proved that the path (17) satisfies the conditions $\left[x(t) x^{\prime}(t)\right]=\sqrt{c(t)}$ and $Q(x)=a(t)$.

In the case $(j j)$, we have $\left[x(t) x^{\prime}(t)\right]=-\sqrt{c(t)}$ and $Q(x)=a(t)$. Then, using Theorem 4.1, where $b(t)=-\sqrt{c(t)})$, we obtain the form (17). As in Theorem 4.1, it is proved that the path (17) satisfies the conditions $\left[x(t) x^{\prime}(t)\right]=-\sqrt{c(t)}$ and $Q(x)=a(t)$.

Theorem 4.5. (i) $A$ path $x(t)$ is an L-non-degenerate $T$-path in $E_{2}$ if and only if the inequality $<x(t), x(t)><x^{\prime}(t), x^{\prime}(t)>-<x(t), x^{\prime}(t)>^{2}>0$ holds for all $t \in T$.

(ii) Let $t_{0} \in T, a(t)$ be a real $C^{(1)}$-function on $T$ such that $a(t)>0$ for all $t \in T$ and $d(t)$ be a continuous function on $T$ such that $a(t) d(t)-$ $\frac{1}{4}\left(a^{\prime}(t)\right)^{2}>0$ for all $t \in T$. Then there exists an L-non-degenerate 
T-path $x(t)$ in $E_{2}$ such that

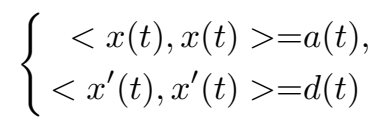

for all $t \in T$. Moreover, a general solution of this system of equations has the following form

$$
x(t)=e^{i \varphi} \sqrt{a(t)} e^{i \int_{t_{0}}^{t} \frac{\sqrt{a(u) d(u)-\frac{1}{4}\left(a^{\prime}(u)\right)^{2}}}{a(u)} d u}
$$

or the following form

$$
x(t)=e^{i \varphi} \sqrt{a(t)} e^{-i \int_{t_{0}}^{t} \frac{\sqrt{a(u) d(u)-\frac{1}{4}\left(a^{\prime}(u)\right)^{2}}}{a(u)} d u},
$$

where $\varphi \in[0,2 \pi)$. Conversely, every T-paths of the forms (19) and (20) are L-non-degenerate T-paths in $E_{2}$ such that the equalities (18) hold.

Proof. (i) Using (12), we obtain that $\left[x(t) x^{\prime}(t)\right]^{2}>0$ for all $t \in T$ if and only if $<x(t), x(t)><x^{\prime}(t), x^{\prime}(t)>-<x(t), x^{\prime}(t)>^{2}>0$ hold for all $t \in T$.

(ii) Assume that a regular $T$-path $x(t)$ in $E_{2}$ such that the equalities (18) hold. Prove that $x(t)$ has the form (19) or (20).

The equality $\langle x(t), x(t)\rangle=a(t)$ for all $t \in T$ implies $\left\langle x(t), x^{\prime}(t)\right\rangle=$ $\frac{1}{2} a^{\prime}(t)$. Put $c(t)=a(t) d(t)-\frac{1}{4}\left(a^{\prime}(t)\right)^{2}$. Using $\langle x(t), x(t)\rangle=a(t)$, $<x(t), x^{\prime}(t)>=\frac{1}{2} a^{\prime}(t),<x^{\prime}(t), x^{\prime}(t)>=d(t)$, the equality (12) and the equality $c(t)=a(t) d(t)-\frac{1}{4}\left(a^{\prime}(t)\right)^{2}$, we obtain that $\left[x(t) x^{\prime}(t)\right]^{2}=c(t)>0$ for all $t \in T$. Hence $x(t)$ is an $L$-non-degenerate $T$-path. Then, by Theorem 4.4, $x(t)$ has the form (16) or (17), where $c(t)=a(t) d(t)-$ $\frac{1}{4}\left(a^{\prime}(t)\right)^{2}$. This means that $x(t)$ has the form (19) or (20).

Conversely, assume that $x(t)$ is the $T$-path of form (19) or (20). Using the equality (12), the equality $c(t)=a(t) d(t)-\frac{1}{4}\left(a^{\prime}(t)\right)^{2}>0$ for all $t \in T$ and Theorem 4.4, we obtain that $x(t)$ is an $L$-non-degenerate $T$-path such that $\langle x(t), x(t)\rangle=a(t)$ and $\left[x(t) x^{\prime}(t)\right]^{2}=c(t)$, where $c(t)=a(t) d(t)-\frac{1}{4}\left(a^{\prime}(t)\right)^{2}$. Using the equality (12), the equalities $c(t)=$ $\left.a(t) d(t)-\frac{1}{4}\left(a^{\prime}(t)\right)^{2},<x(t), x^{\prime}(t)\right\rangle=\frac{1}{2} a^{\prime}(t)$ and $\langle x(t), x(t)\rangle=a(t)>0$ for all $t \in T$, we obtain $\left\langle x^{\prime}(t), x^{\prime}(t)\right\rangle=d(t)$. 


\section{The type and invariant parametrizations of an $L$-non- degenerate curve}

Some definitions on curves are given in the section "Introduction".

Proposition 5.1. Let $\alpha$ be an L-regular curve. Then every parametrization $x \in \alpha$ is an L-regular path.

Proof. Let $\alpha$ be an L-regular curve. Then there exists a parametrization $x \in \alpha$ such that $x(t)$ is an L-regular $J$-path, where $J=(a, b) \subseteq R$. Let $y \in \alpha$ be an arbitrary parametrization and $y(u)$ is an $U$-path, where $U=(c, d) \subseteq R$. Then there exists a $C^{(1)}$-diffeomorphism $\varphi: U \rightarrow J$ such that $\varphi^{\prime}(r)>0$ and $y(r)=x(\varphi(r))$ for all $r \in U$. Since $x(t)$ is an L-regular $J$-path, $<x(t), x(t)>\neq$ 0 for all $t \in J$. We have $<y(r), y(r)>=<x(\varphi(r)), x(\varphi(r))>$. Since $<$ $x(t), x(t)>\neq 0$ for all $t \in J$, we have $<y(r), y(r)>=<x(\varphi(r)), x(\varphi(r))>\neq 0$ for all $r \in U$. Hence $y(r)$ is an L-regular path.

Definition 5.2. A curve $\alpha$ will be called L-non-degenerate if it contains an L-non-degenerate path.

Proposition 5.3. Let $\alpha$ be an L-non-degenerate curve. Then every parametrization $x \in \alpha$ is an L-non-degenerate path.

Proof. Let $\alpha$ be an L-non-degenerate curve. Then there exists a parametrization $x \in \alpha$ such that $x(t)$ is an L-non-degenerate $J$-path, where $J=(a, b) \subseteq R$. Let $y \in \alpha$ be an arbitrary parametrization and $y(u)$ is an $U$-path, where $U=(c, d) \subseteq R$. Then there exists a $C^{(1)}$-diffeomorphism $\varphi: U \rightarrow J$ such that $\varphi^{\prime}(r)>0$ and $y(r)=x(\varphi(r))$ for all $r \in U$. Since $x(t)$ is an L-non-degenerate $J$-path, $\left[x(t) x^{\prime}(t)\right] \neq 0$ for all $t \in J$. We have

$$
\begin{aligned}
{\left[y(r) y^{\prime}(r)\right]=\left[x(\varphi(r)) \frac{d x(\varphi(r))}{d r}\right]=} & {\left[x(\varphi(r))\left(\varphi^{\prime}(r) \frac{d x(\varphi(r))}{d \varphi}\right)\right]=} \\
& =\varphi^{\prime}(r)\left[x(\varphi(r)) \frac{d x(\varphi(r))}{d \varphi}\right] .
\end{aligned}
$$

This equality and the inequality $\left[x(t) x^{\prime}(t)\right] \neq 0$ for all $t \in J$ implies the inequality

$$
\begin{aligned}
& {\left[x(\varphi(r)) \frac{d x(\varphi(r))}{d \varphi}\right] \neq 0 \text { for all } r \in U \text {. Since } \varphi^{\prime}(r)>0 \text { for all } r \in U \text { and }} \\
& {\left[x(\varphi(r)) \frac{d x(\varphi(r))}{d \varphi}\right] \neq 0 \text { for all } r \in U, \text { the above equality implies }\left[y(r) y^{\prime}(r)\right] \neq 0} \\
& \text { for all } r \in U \text {. Hence } y(r) \text { is an L-non-degenerate path. }
\end{aligned}
$$

Now we define invariant parametrizations of L-non-degenerate curves in $E_{2}$. Let $x(t)$ be an L-non-degenerate $J$-path in $E_{2}$, where $J=(a, b) \subseteq R, a<b$. 
For $c, d \in R$ such that $a<c<d<b$, we put $l_{x}(c, d)=\int_{c}^{d}\left|\left[x(t) x^{\prime}(t)\right]\right| d t$. The limits $l_{x}(a, d)=\lim _{c \rightarrow a} l_{x}(c, d) \leq+\infty$ and $l_{x}(c, b)=\lim _{d \rightarrow b} l_{x}(c, d) \leq+\infty$ exist. There are only four possibilities:

$\left(\gamma_{1}\right) 0<l_{x}(a, d)<+\infty, 0<l_{x}(c, b)<+\infty$;

$\left(\gamma_{2}\right) 0<l_{x}(a, d)<+\infty, l_{x}(c, b)=+\infty ;$

$\left(\gamma_{3}\right) l_{x}(a, d)=+\infty, 0<l_{x}(c, b)<+\infty ;$

$\left(\gamma_{4}\right) l_{x}(a, d)=+\infty, l_{x}(c, b)=+\infty$

Suppose that the case $\left(\gamma_{1}\right)$ or $\left(\gamma_{2}\right)$ holds for some $c, d \in J$. Then $l=$ $l_{x}(a, d)+l_{x}(c, b)-l_{x}(c, d)$, where $0 \leq l \leq+\infty$, does not depend on $c, d \in J$. In this case we say that $x$ belongs to the $O(2)$-type of $(0, l)$. The cases $\left(\gamma_{3}\right)$ and $\left(\gamma_{4}\right)$ do not depend on $c, d$. In these cases, we say that $x$ belongs to the $O(2)$-types of $(-\infty, 0)$ and $(-\infty,+\infty)$, respectively. There exist paths of all $O(2)$-types $(0, l)$, where $l<+\infty,(0,+\infty),(-\infty, 0)$, and $(-\infty,+\infty)$. The $O(2)$-type of a path $x$ will be denoted by $T L(x)$.

Proposition 5.4. (i) Let $x$ and $y$ be L-non-degenerate paths in $E_{2}$. If $x \stackrel{O(2)}{\sim} y$, then $T L(x)=T L(y) ;$

(ii) If $x$ and $y$ are parametrizations of an L-non-degenerate curve $\alpha$, then $T L(x)=T L(y)$.

Proof. A proof is easy and it is omitted.

The $O(2)$-type of a path $x \in \alpha$ will be called the $O(2)$-type of the nondegenerate curve $\alpha$ and denoted by $T L(\alpha)$.

Proposition 5.5. Let $\alpha$ and $\beta$ be non-degenerate curves in $E_{2}$. If $\alpha \stackrel{O(2)}{\sim} \beta$, then $T L(\alpha)=T L(\beta)$.

Proof. A proof is easy and it is omitted.

Now we define an invariant parametrization of an L-non-degenerate curve in $E_{2}$. Let $J=(a, b)$ and $x(t)$ be an L-non-degenerate $J$-path in $E_{2}$. We define the $O(2)$-type arc length function $s_{x}(t)$ for each $O(2)$-type as follows. We put $s_{x}(t)=l_{x}(a, t)$ for the case $T L(x)=(0, l)$, where $l \leq+\infty$, and $s_{x}(t)=-l_{x}(t, b)$ for the case $T L(x)=(-\infty, 0)$. 
Assume that $T L(x)=(-\infty,+\infty)$. We define the function $s_{x}(t)$ for this case. We choose a fixed point in every interval $J=(a, b)$ of $R$ and denote it by $a_{J}$. Put $a_{J}=0$ for $J=(-\infty,+\infty)$. For the $J$-path $x(t)$, we set $s_{x}(t)=l_{x}\left(a_{J}, t\right)$.

Since $s_{x}^{\prime}(t)>0$ for all $t \in J$, the inverse function of $s_{x}(t)$ exists. Let us denote it by $t_{x}(s)$. The domain of $t_{x}(s)$ is $T L(x)$ and $t_{x}^{\prime}(s)>0$ for all $s \in T L(x)$.

Proposition 5.6. Let $U=(a, b)$ and $x$ be an L-non-degenerate $U$-path in $E_{2}$ and $J=(c, d) \subseteq R$. Then:

(i) $s_{F x}(t)=s_{x}(t), \forall t \in U$ and $t_{F x}(s)=t_{x}(s), \forall s \in T L(x)$, for all $F \in O(2)$;

(ii) the equalities $s_{x(\varphi)}(r)=s_{x}(\varphi(r))+s_{0}, \forall r \in J$ and $\varphi\left(t_{x(\varphi)}\left(s+s_{0}\right)\right)=$ $t_{x}(s), \forall s \in T L(x)$ hold for any $C^{(1)}$-diffeomorphism $\varphi: J=(c, d) \rightarrow U$ such that $\varphi^{\prime}(r)>0$ for all $r \in J$, where $s_{0}=0$ for $T L(x) \neq(-\infty,+\infty)$ and $s_{0}=l_{x}\left(\varphi\left(a_{J}\right), a_{U}\right)$ for $T L(x)=(-\infty,+\infty)$.

Proof. It is similar to the proof of Proposition 2 in [4].

Let $\alpha$ be an L-non-degenerate curve, $x \in \alpha$. Then $x\left(t_{x}(s)\right)$ is a parametrization of $\alpha$.

Definition 5.7. (see $[4,15])$ The parametrization $x\left(t_{x}(s)\right)$ of an L-nondegenerate curve $\alpha$ will be called an invariant parametrization of $\alpha$.

We denote the set of all invariant parametrizations of an L-non-degenerate $\alpha$ by $\operatorname{Ip}(\alpha)$. Every $y \in I p(\alpha)$ is a $J$-path, where $J=T L(\alpha)$.

Proposition 5.8. Let $\alpha$ be an L-non-degenerate curve, $x \in \alpha$ and $x$ be a $J$-path, where $J=T L(\alpha)$. Then the following conditions are equivalent:

(i) $x$ is an invariant parametrization of $\alpha$;

(ii) $\left|\left[x(s) x^{\prime}(s)\right]\right|=1$ for all $s \in T L(\alpha)$;

(iii) $s_{x}(s)=s$ for all $s \in T L(\alpha)$.

Proof. It is similar to the proof of Proposition 3 in [4].

Below $J \subseteq R$, where $J$ is a one of sets $(0, l), l<+\infty ;(0,+\infty),(-\infty, 0)$ or $(-\infty,+\infty)$.

Theorem 5.9. Let $\alpha$ be an L-non-degenerate curve in $E_{2}$ and $x(s) \in I p(\alpha)$. Then $x(s)$ has the following form

$$
x(s)=e^{i \varphi} \sqrt{a(s)} e^{i \int_{s_{0}}^{s} \frac{1}{a(u)} d u},
$$


or the following form

$$
x(s)=e^{i \varphi} \sqrt{a(s)} e^{-i \int_{s_{0}}^{s} \frac{1}{a(u)} d u},
$$

where $\varphi \in[0,2 \pi)$ and $a(s)$ is a real continuous functions on $J$.

Conversely, every path $x(s)$ of the form (21) or the form (22) is an invariant parametrization of an L-non-degenerate curve in $E_{2}$.

Proof. Let $\alpha$ be an L-non-degenerate curve in $E_{2}$ and $x(s) \in I p(\alpha)$. Then, by Proposition 5.8(ii), $\left|\left[x(s) x^{\prime}(s)\right]\right|=1, \forall s \in T L(\alpha)$. Put $c(s)=\left[x(s) x^{\prime}(s)\right]^{2}$ and $a(s)=<x(s), x(s)>$ for all $s \in T L(\alpha)$. Then $c(s)=1$ for all $s \in T L(\alpha)$. This equality, equalities (16) and (17) imply that $x(s)$ has the form (21) in the case $\left[x(s) x^{\prime}(s)\right]=1, \forall s \in T L(\alpha)$ and the form (22) in the case $\left[x(s) x^{\prime}(s)\right]=$ $-1, \forall s \in T L(\alpha)$.

Conversely, assume that $x(s)$ has the form (21) or the form (22). Then, by Theorem 4.4, $Q(x(s))=a(s)$ and $\left|\left[x(s) x^{\prime}(s)\right]\right|^{2}=c(s)=1$. By Proposition $5.8(i i)$, the equality $\left|\left[x(s) x^{\prime}(s)\right]\right|^{2}=1$ implies that the path $x(s)$ is an invariant parametrization.

Proposition 5.10. Let $\alpha$ be an L-non-degenerate curve and $T L(\alpha) \neq(-\infty,+\infty)$. Then there exists the unique invariant parametrization of $\alpha$.

Proof. A proof is similar to the proof of Proposition 4 in [4].

Let $\alpha$ be an L-non-degenerate curve and $L(\alpha)=(-\infty,+\infty)$. Then it is easy to see that the set $\operatorname{Ip}(\alpha)$ is infinite and it is not countable.

Remark 5.11. We note that if $x(t)$ is a periodic L-non-degenerate path then $T L(x)=(-\infty,+\infty)$.

Proposition 5.12. Let $\alpha$ be an L-non-degenerate curve, $T L(\alpha)=(-\infty,+\infty)$ and $x \in \operatorname{Ip}(\alpha)$. Then $\operatorname{Ip}(\alpha)=\{y: y(s)=x(s+c), c \in(-\infty,+\infty)\}$.

Proof. A proof is similar to the proof of Proposition 5 in [4].

Let $H$ be a one of groups $O(2), O^{+}(2)$.

Theorem 5.13. Let $\alpha, \beta$ be L-non-degenerate curves and $x \in I p(\alpha), y \in$ Ip $(\beta)$. Then:

(i) for $T L(\alpha)=T L(\beta) \neq(-\infty,+\infty), \alpha \stackrel{H}{\sim} \beta$ if and only if $x \stackrel{H}{\sim} y$;

(ii) for $T L(\alpha)=T L(\beta)=(-\infty,+\infty), \alpha \stackrel{H}{\sim} \beta$ if and only if $x \stackrel{H}{\sim} y\left(\psi_{c}\right)$ for some $c \in(-\infty,+\infty)$, where $\psi_{c}(s)=s+c$. 
Proof. A proof of this theorem for the group $H$ is similar to the proof of Theorem 1 in [4] and it is omitted.

Theorem 5.13 reduces the problem of the $H$-equivalence of L-non-degenerate curves for groups $O(2), O^{+}(2)$ to that of paths only for the case $T L(\alpha)=$ $T L(\beta) \neq(-\infty,+\infty)$.

Definition 5.14. Let $J=(-\infty,+\infty)$. J-paths $x(t)$ and $y(t)$ will be called $[H,(-\infty,+\infty)]$-equivalent, if there exist $h \in H$ and $d \in(-\infty,+\infty)$ such that $y(t)=h x(t+d)$ for all $t \in J$.

Let $\alpha, \beta$ be L-non-degenerate curves such that $T L(\alpha)=T L(\beta)=(-\infty,+\infty)$. In this case, Theorem 5.13 reduces the problem of the $H$-equivalence of these curves to the problem of $[H,(-\infty,+\infty)]$-equivalence of paths.

\section{Complete systems of invariants of an L-non-degenerate curve and uniqueness theorems}

By Theorem 5.13, G-equivalence and uniqueness problems for curves reduce to the same problems for invariant parametrizations of curves only for the case $T L(\alpha)=T L(\beta) \neq(-\infty,+\infty)$. Below we use this reduction.

Theorem 6.1. Let $\alpha, \beta$ be L-non-degenerate curves in $E_{2}, T L(\alpha) \neq(-\infty,+\infty)$, $T L(\beta) \neq(-\infty,+\infty)$ and $x \in \operatorname{Ip}(\alpha), y \in \operatorname{Ip}(\beta)$.

(i) Assume that $\alpha \stackrel{O^{+}(2)}{\sim} \beta$. Then, for all $s \in T L(\alpha)$ the following equalities hold

$$
\left\{\begin{aligned}
T L(\alpha) & =T L(\beta) \\
<x(s), x(s)> & =<y(s), y(s)> \\
\operatorname{Sgn}\left(\left[x(s) x^{\prime}(s)\right]\right) & =\operatorname{Sgn}\left(\left[y(s) y^{\prime}(s)\right]\right) .
\end{aligned}\right.
$$

(ii) Conversely, assume that the equalities (23) hold. Then there exists the unique $F \in O^{+}(2)$ such that $\beta=F \alpha$. In this case, $F=U$, where $U \in O^{+}(2), U$ has the following form

$$
U=\left(\begin{array}{cc}
\frac{\langle x(s), y(s)>}{Q(x(s))} & -\frac{[x(s) y(s)]}{Q(x(s))} \\
\frac{[x(s) y(s)]}{Q(x(s))} & \frac{\leq x(s), y(s)>}{Q(x(s))}
\end{array}\right)
$$

and the matrix $U$ does not depend on $s \in T L(\alpha)$. 
Proof. (i) Let $\alpha \stackrel{O^{+}(2)}{\sim} \beta$. By Proposition 5.5, we have $T L(\alpha)=T L(\beta)$. This equality, $\alpha \stackrel{O^{+}(2)}{\sim} \beta$ and Theorem 5.13(i) imply $x \stackrel{O^{+}(2)}{\sim} y$. By Theorem 3.2, $x \stackrel{O^{+}(2)}{\sim} y$ implies the equalities (3). By the equalities (3), the equalities $\left\langle x(s), x(s)>=<y(s), y(s)>\right.$ and $\left[x(s) x^{\prime}(s)\right]=$ $\left[y(s) y^{\prime}(s)\right]$ hold for all $s \in T L(\alpha)$. The equality $\left[x(s) x^{\prime}(s)\right]=\left[y(s) y^{\prime}(s)\right]$ implies the equality

$\operatorname{Sgn}\left(\left[x(s) x^{\prime}(s)\right]\right)=\operatorname{Sgn}\left(\left[y(s) y^{\prime}(s)\right]\right)$ for all $s \in T L(\alpha)$. Hence equalities (23) hold.

(ii) Conversely, assume that the equalities (23) hold. Since $x(s)$ and $y(s)$ are invariant parametrizations, by Proposition 5.8(ii), we have $\left|\left[x(s) x^{\prime}(s)\right]\right|=$ 1 and $\left|\left[y(s) y^{\prime}(s)\right]\right|=1$ for all $s \in T L(\alpha)$. These equalities imply $\left|\left[x(s) x^{\prime}(s)\right]\right|=\left|\left[y(s) y^{\prime}(s)\right]\right|$ for all $s \in T L(\alpha)$. This equality and the equality $\operatorname{Sgn}\left(\left[x(s) x^{\prime}(s)\right]\right)=\operatorname{Sgn}\left(\left[y(s) y^{\prime}(s)\right]\right)$ imply the equality $\left[x(s) x^{\prime}(s)\right]=$ $\left[y(s) y^{\prime}(s)\right]$ for all $s \in T L(\alpha)$. This equality and the equalities (23) imply

the equalities (3). By Theorem 3.2, these equalities imply $x \stackrel{O^{+}(2)}{\sim} y$ and there exists the unique $F \in O^{+}(2)$ such that $y(s)=F x(s)$. In this case, $F=U$, where $U \in O^{+}(2), U$ has the form (24). Here, the matrix $U$ does not depend on $s \in T L(\alpha)$. Using $x \in \operatorname{Ip}(\alpha), y \in \operatorname{Ip}(\beta)$, Theorem 5.13(i) and $y(s)=F x(s)$ for all $s \in T L(\alpha)$, we obtain that $\beta=F \alpha$.

Remark 6.2. By Theorem 6.1, the system $\left\{T L(\alpha),<x(s), x^{\prime}(s)>, \operatorname{Sgn}\left(\left[x(s) x^{\prime}(s)\right]\right)\right\}$, where $x(s)$ is the invariant parametrization of the curve $\alpha$, is a complete system of $O^{+}$-invariants of a curve $\alpha$ for the case $T L(\alpha) \neq(-\infty,+\infty)$. But they are not $O^{+}(2)$-invariants of a curve $\alpha$, in the case $T L(\alpha)=(-\infty,+\infty)$.

Theorem 6.3. Let $\alpha, \beta$ be L-non-degenerate curves in $E_{2}, T L(\alpha)=T L(\beta)=$ $(-\infty,+\infty)$ and $x \in \operatorname{Ip}(\alpha), y \in \operatorname{Ip}(\beta)$.

(i) Assume that $\alpha \stackrel{O^{+}(2)}{\sim} \beta$. Then there exists $s_{1} \in(-\infty,+\infty)$ such that the following equalities hold for all $s \in(-\infty,+\infty)$

$$
\left\{\begin{aligned}
<x\left(s+s_{1}\right), x^{\prime}\left(s+s_{1}\right)> & =<y(s), y^{\prime}(s)>, \\
\operatorname{Sgn}\left(\left[x(s) x^{\prime}(s)\right]\right) & =\operatorname{Sgn}\left(\left[y^{\prime}(s) y^{\prime}(s)\right]\right) .
\end{aligned}\right.
$$

(ii) Conversely, assume that the equalities (25) hold for some $s_{1} \in(-\infty,+\infty)$ and all $s \in(-\infty,+\infty)$. Then there exists the unique $F \in O^{+}(2)$ such 
that $\beta=F \alpha$. In this case, $F=U$, where $U \in O^{+}(2), U$ has the following form

$$
U=\left(\begin{array}{cc}
\frac{\left\langle x\left(s+s_{1}\right), y(s)>\right.}{Q\left(x\left(s+s_{1}\right)\right)} & -\frac{\left[x\left(s+s_{1}\right) y(s)\right]}{Q\left(x\left(s+s_{1}\right)\right)} \\
\frac{\left[x\left(s+s_{1}\right) y(s)\right]}{Q\left(x\left(s+s_{1}\right)\right)} & \frac{\left.<x\left(s+s_{1}\right), y(s)\right\rangle}{Q\left(x\left(s+s_{1}\right)\right)}
\end{array}\right),
$$

and the matrix $U$ does not depend on $s \in T L(\alpha)$.

Proof. (i) Let $\alpha \stackrel{O^{+}(2)}{\sim} \beta$. By the supposition in this theorem, we have $T L(\alpha)=T L(\beta)=(-\infty,+\infty)$. By this equality, $\alpha \stackrel{O^{+}(2)}{\sim} \beta$ and Theorem $5.13(i i)$, there exists $s_{1} \in(-\infty,+\infty)$ such that $x\left(s+s_{1}\right) \stackrel{O^{+}(2)}{\sim} y(s)$. By Theorem 3.2(i), $x\left(s+s_{1}\right) \stackrel{O^{+}(2)}{\sim} y(s)$ implies the equalities (3) for $x\left(s+s_{1}\right)$ and $y(s)$. That is,

$$
\left\{\begin{aligned}
<x\left(s+s_{1}\right), x^{\prime}\left(s+s_{1}\right)> & =<y(s), y^{\prime}(s)>, \\
{\left[x\left(s+s_{1}\right) x^{\prime}\left(s+s_{1}\right)\right] } & =\left[y(s) y^{\prime}(s)\right] .
\end{aligned}\right.
$$

Hence the equality $<x\left(s+s_{1}\right), x^{\prime}\left(s+s_{1}\right)>=<y(s), y^{\prime}(s)>$ holds for all $s \in T L(\alpha)$. The equality $\left[x\left(s+s_{1}\right) x^{\prime}\left(s+s_{1}\right)\right]=\left[y(s) y^{\prime}(s)\right]$ implies the equality

$\operatorname{Sgn}\left(\left[x(s) x^{\prime}(s)\right]\right)=\operatorname{Sgn}\left(\left[x\left(s+s_{1}\right) x^{\prime}\left(s+s_{1}\right)\right]\right)=\operatorname{Sgn}\left(\left[y(s) y^{\prime}(s)\right]\right)$ for all $s \in T L(\alpha)$. Hence the equalities (25) hold.

(ii) Conversely, assume that the equalities (25) hold for some $s_{1} \in(-\infty,+\infty)$ and all $s \in(-\infty,+\infty)$. Since $x(s), y(s)$ are invariant parametrizations, by Proposition 5.8(ii), we have $\left|\left[x(s) x^{\prime}(s)\right]\right|=1$ and $\left|\left[y(s) y^{\prime}(s)\right]\right|=1$ for all $s \in T L(\alpha)$. These equalities imply $\left|\left[x\left(s+s_{1}\right) x^{\prime}\left(s+s_{1}\right)\right]\right|=$ $\left|\left[x(s) x^{\prime}(s)\right]\right|=\left|\left[y(s) y^{\prime}(s)\right]\right|$ for all $s \in T L(\alpha)$. These equalities and $\operatorname{Sgn}\left(\left[x(s) x^{\prime}(s)\right]\right)=\operatorname{Sgn}\left(\left[y(s) y^{\prime}(s)\right]\right)$ imply the equality $\left[x\left(s+s_{1}\right) x^{\prime}\left(s+s_{1}\right)\right]=\left[y(s) y^{\prime}(s)\right]$ for all $s \in T L(\alpha)$. This equality and the equalities (25) imply the equalities

$$
\left\{\begin{aligned}
<x\left(s+s_{1}\right), x^{\prime}\left(s+s_{1}\right)> & =<y(s), y^{\prime}(s)>, \\
{\left[x\left(s+s_{1}\right) x^{\prime}\left(s+s_{1}\right)\right] } & =\left[y(s) y^{\prime}(s)\right]
\end{aligned}\right.
$$

for all $s \in T L(\alpha)$. By Theorem 3.2, $x\left(s+s_{1}\right) \stackrel{O^{+}(2)}{\sim} y(s)$ and there exists the unique $F \in O^{+}(2)$ such that $y(s)=F x\left(s+s_{1}\right)$ for all $s \in T L(\alpha)$. In this case, $F=U$, where $U \in O^{+}(2), U$ has the form (26). Here, the matrix $U$ does not depend on $s \in T L(\alpha)$. Using $x \in I p(\alpha), y \in I p(\beta)$, 
Theorem 5.13(ii) and $y(s)=F x\left(s+s_{1}\right)$ for all $s \in T L(\alpha)$, we obtain that $\beta=F \alpha$.

Theorem 6.4. Let $\alpha, \beta$ be L-non-degenerate curves in $E_{2}, T L(\alpha) \neq(-\infty,+\infty)$, $T L(\beta) \neq(-\infty,+\infty)$ and $x \in \operatorname{Ip}(\alpha), y \in \operatorname{Ip}(\beta)$.

(i) Assume that $\alpha \stackrel{O(2)}{\sim} \beta$. Then we have

$$
\left\{\begin{array}{c}
T L(\alpha)=T L(\beta), \\
<x(s), x^{\prime}(s)>=<y(s), y^{\prime}(s)>
\end{array}\right.
$$

for all $s \in T L(\alpha)$.

(ii) Conversely, assume that the equalities (27) hold. Then $\alpha \stackrel{O(2)}{\sim} \beta$. Moreover, the unique $F \in O(2)$ exists such that $\beta=F \alpha$. In this case, only two following cases exist:

(ii.1) $\operatorname{Sgn}\left(\left[x(s) x^{\prime}(s)\right]\right)=\operatorname{Sgn}\left(\left[y(s) y^{\prime}(s)\right]\right)$;

(ii.2) $\operatorname{Sgn}\left(\left[x(s) x^{\prime}(s)\right]\right)=-\operatorname{Sgn}\left(\left[y(s) y^{\prime}(s)\right]\right)$.

In the case (ii.1), $F=U_{1}$, where $U_{1}$ has the form (26).

In the case (ii.2), $F=\left(U_{2} W\right)$, where $U_{2}$ has the following form

$$
U_{2}=\left(\begin{array}{cc}
\frac{\langle W x(s) y(s)>}{Q(W x(s))} & -\frac{[(W x(s)) y(s)]}{Q(W x(s))} \\
\frac{[(W x(s) y(s)]}{Q(W x(s))} & \frac{\langle W x(s), y(s)>}{Q(W x(s))}
\end{array}\right),
$$

Here, the matrices $U_{1}$ and $U_{2}$ do not depend on $s \in T L(\alpha)$.

Proof. A proof follows easy from Theorems 5.13(i), 3.9 and 6.1.

Theorem 6.5. Let $\alpha, \beta$ be L-non-degenerate curves in $E_{2}, T L(\alpha)=T L(\beta)=$ $(-\infty,+\infty)$ and $x \in \operatorname{Ip}(\alpha), y \in \operatorname{Ip}(\beta)$.

(i) Assume that $\alpha \stackrel{O(2)}{\sim} \beta$. Then there exists $s_{1} \in(-\infty,+\infty)$ such that the following equality

$$
<x\left(s+s_{1}\right), x^{\prime}\left(s+s_{1}\right)>=<y(s), y^{\prime}(s)>
$$

holds for all $s \in(-\infty,+\infty)$. 
(ii) Conversely, assume that there exists $s_{1} \in(-\infty,+\infty)$ such that the equality (28) holds for all $s \in(-\infty,+\infty)$. Then $\alpha \stackrel{O(2)}{\sim} \beta$. Moreover, $F \in O(2)$ exists such that $\beta=F \alpha$. In this case, following cases exist:

(ii.1) $\left[x\left(s+s_{1}\right) x^{\prime}\left(s+s_{1}\right)\right]=\left[y(s) y^{\prime}(s)\right]$;

(ii.2) $\left[x\left(s+s_{1}\right) x^{\prime}\left(s+s_{1}\right)\right]=-\left[y(s) y^{\prime}(s)\right]$.

In the case (ii.1), $F=U_{1}$, where $U_{1}$ has the form (26).

In the case (ii.2), $F=\left(U_{2} W\right)$, where $U_{2}$ has the following form

$$
U_{2}=\left(\begin{array}{cc}
\frac{\left\langle W x\left(s+s_{1}\right), y(s)>\right.}{Q\left(W x\left(s+s_{1}\right)\right)} & -\frac{\left[W x\left(s+s_{1}\right) y(s)\right]}{Q\left(W x\left(s+s_{1}\right)\right)} \\
\frac{\left[W x\left(s+s_{1}\right) y(s)\right]}{Q\left(W x\left(s+s_{1}\right)\right)} & \frac{\left\langle W x\left(s+s_{1}\right), y(s)>\right.}{Q\left(W x\left(s+s_{1}\right)\right)}
\end{array}\right) .
$$

Here, the matrices $U_{1}$ and $U_{2}$ do not depend on $s \in T L(\alpha)$.

Proof. A proof follows easy from Theorems 5.13(ii), 3.9 and 6.3.

\subsection{Conclusion}

Methods, developed in the present work, will be useful in the theory of global invariants of curves in two-dimensional geometries and physics. This approach is also will be useful in the theory of global invariants of vector fields and a system of curves in two-dimensional geometries and physics.

\section{Acknowledgements}

The authors is very grateful to the reviewer(s) for helpful comments and valuable suggestions.

This work is supported by the Scientific and Technological Research Council of Turkey, (Grant No:1059B211601787)

\section{References}

[1] Alcázar, J. G., Hermoso, Carlos, M., Muntingh,G., Detecting similarity of rational plane curves. J. Comput. Appl. Math 269 (2014), 113. 
[2] Alexandrov, A. D., Reshetnyak, Yu. G., General Theory of Irregular Curves. Kluwer Acad. Publ.,Dordrecht, 1989.

[3] Aminov, Yu., Differential Geometry and Topology of Curves. CRC Press, New York, 2000.

[4] Aripov, R. G., Khadjiev (Khadzhiev) D., The complete system of global differential and integral invariants of a curve in Euclidean geometry. Russian Mathematics (Iz VUZ), 51(7),(2007) 1-14.

[5] Berger, M., Geometry I. Springer-Verlag, Berlin Heidelberg, 1987.

[6] Chou, K. S., Qu, C. Z., Integrable equations arising from motions of plane curves. Phys. D 162(1-2) (2002) 9-33.

[7] Chou, K. S., Qu, C. Z., Integrable equations and motions of plane curves. Symmetry in nonlinear mathematical physics, Part 1, 2 Kyiv, (2001) 281290.

[8] Gibson, G. G., Elementary Geometry of Differentiable Curves. An Undergraduate Introduction. Cambridge University Press, 2001.

[9] Guggenheimer, H., W., Differential Geometry. McGraw-Hill Book Company, Inc., New York, 1963.

[10] Hauer, M., Jüttler, B. Detecting affine equivalences of planar rational curves. EuroCG 2016, Lugano, Switzerland, March 30-April 1, 2016.

[11] Inoguchi, J., Attractive plane curves in differential geometry. Mathematical Progress in Expressive Image Synthesis III, (2016) 121-135.

[12] Khadjiev, D., Application of the Invariant Theory to the Differential Geometry of Curves. Fan Publisher, Tashkent, 1988. [in Russian].

[13] Khadjiev, D., Complete systems of differential invariants of vector fields in a Euclidean space. Turk J. Math., 34, (2010) 543-560.

[14] Khadjiev, D., On invariants of immersions of an n-dimensional manifold in an n-dimensional pseudo-Euclidean space. J Nonlinear Math Phys, 17, Supp 01 (2010) 49-70.

[15] Khadjiev D., Peksen Ö., The complete system of global differential and integral invariants of equiaffne curves. Diff. Geom. And Appl., 20 (2004) 168-175. 
[16] Khadjiev, D., Ören, İ., Pekşen, Ö., Generating systems of differential invariants and the theorem on existence for curves in the pseudo-Euclidean geometry. Turk J. Math., 37 (2013) 80-94.

[17] Kreyszig, E., Introduction to Differential Geometry and Riemannian Geometry. Univ. of Toronto Press, 1968.

[18] Mozo-Fernandez, J., Munuera,C., Recognition of polynomial plane curves under affine transformations. AAECC 13 (2002) 121-136.

[19] O’Neill, B., Elementary Differential Geometry, Elsevier, 2006.

[20] Ören, İ., Equivalence conditions of two Bézier curves in the Euclidean geometry. Iran J Sci Technol Trans Sci. DOI number: 10.1007/s40995016-0129-1.(in press)

[21] Pekşen, Ö., Khadjiev, D., On invariants of null curves in the pseudoEuclidean geometry. Diff. Geom. And Appl. 29 (2011) S183-187.

[22] Pekşen, Ö., Khadjiev, D., Ören,İ, Invariant parametrizations and complete systems of global invariants of curves in the pseudo-Euclidean geometry. Turk J. Math., 36 (2012) 147-160.

[23] Yaglom, I. M., Complex Numbers in Geometry. Academic Press, New York, 1968.

[24] Zwikker, C., The Advanced Geometry of Plane Curves and Their Applications. Dover Publications, INC., New York, 1963.

Djavvat KHADJIEV,

Institute of Mathematics,

Academy of Sciences of Uzbekistan,

100125,Tashkent, Uzbekistan.

Email: khdjavvat@gmail.com

İdris ÖREN,

Department of Mathematics,

Karadeniz Technical University,

61080, Trabzon, Turkey.

Email: oren@ktu.edu.tr 